\title{
Le développement durable entre temps court et temps long, entre principe de gestion et principe de discussion
}

\section{Nicole d'Almeida}

\section{(2) OpenEdition \\ Journals}

Édition électronique

URL : http://journals.openedition.org/communicationorganisation/3393

DOI : 10.4000/communicationorganisation.3393

ISSN : 1775-3546

Éditeur

Presses universitaires de Bordeaux

Édition imprimée

Date de publication : 1 juin 2006

Pagination : 124-138

ISSN : 1168-5549

Référence électronique

Nicole d'Almeida, «Le développement durable entre temps court et temps long, entre principe de gestion et principe de discussion », Communication et organisation [En ligne], 29 | 2006, mis en ligne le 21 juin 2012, consulté le 18 décembre 2020. URL : http://journals.openedition.org/

communicationorganisation/3393; DOI : https://doi.org/10.4000/communicationorganisation.3393

Ce document a été généré automatiquement le 18 décembre 2020.

(c) Presses universitaires de Bordeaux 


\title{
Le développement durable entre temps court et temps long, entre principe de gestion et principe de discussion
}

\author{
Nicole d'Almeida
}

«Vite, vite, de la lenteur », Mozart

1 Le contexte d'émergence du discours du développement durable est marqué par un manque de temps et par un besoin de temps. Le manque de temps est celui de sociétés, d'organisations et d'individus vivant dans l'instant, enfermés dans l'étroitesse du moment, oublieux du passé et incapables d'envisager un avenir. La modernité ou l'hypermodernité sont aujourd'hui définies par un certain nombre d'auteurs un comme rapport particulier au temps, comme un manque de temps ou un excès de temps: $\mathrm{H}$. Arendt montre l'ouverture du règne de la discontinuité lié à la rupture du fil de la tradition tandis que G. Virilio met l'accent sur l'accélération généralisée des sociétés occidentales.

2 Tout ceci rend possible l'installation durable à tous niveaux d'une logique de crise qui rend nécessaire le besoin de retrouver le sens de la durée au sens bergsonien. C'est ainsi que surgit le besoin d'un autre temps, un besoin de lenteur (cf l'éloge de la lenteur de P. Sanson), un besoin d'éternité que les tendances religieuses ou mystiques en plein essor prennent en charge ou encore un besoin de continuité. Ce besoin se retrouve dans la société (où les loisirs) et dans les organisations : stages organisés dans des couvents et lieux de retraite pour cadres comme l'ont imaginé le groupe Auchan et HEC. Retrouver du temps ou le temps c'est se retrouver soi même, retrouver sa capacité de jugement, d'initiatives, de création et c'est sur le ressort temporel qu'interviennent bon nombre de coaches dont la mission n'est finalement autre que l'apprentissage de la décélération. Gestion du temps et management de soi passent par une révision du rapport au temps. 
3 Le capitalisme est aujourd'hui pressé, il ne supporte pas les lenteurs ni les freins, il a cette inédite capacité à dévorer l'espace (à couvrir le champ mondial) et le temps, à remplir l'espace et à disloquer le temps. Dans le monde des affaires comme dans celui des médias, le temps est devenu un facteur fortement différenciant, un nœud essentiel de concurrentialité tant dans les services que dans les autres secteurs où l'important est d'agir vite.

\section{Le Développement Durable et son double statut, idéal au long cours et principe de gestion}

Du temps long au temps court, nous analyserons ici un paradoxe que nous nommerons le double statut du Développement Durable, idéal normatif et principe de gestion, objet de discussion et objet comptable. Le principe du développement durable est à la fois entré dans la sphère publique de la discussion, parfois très vive comme lors du Sommet de Johannesburg et institué par le système économique et financier qui envisage aujourd'hui une réorientation des principes de gestion et d'évaluation de la performance. Ce n'est pas un hasard si c'est l'Ordre des Experts Comptables qui, en France, a créé et décerne chaque année les prix du rapport environnemental (devenu ensuite prix du rapport DD).

La notion de développement durable est devenue une question publique, construite comme publique mais susceptible d'être traitée soit par l'expérimentation politique (le débat enrôlant de nouvelles catégories sociales, source d'un renouvellement possible $\mathrm{du}$ politique et de son objet désormais mis à l'épreuve de la durée) soit par une technique de gestion (une batterie d'indicateurs et de tableaux de bord au service de la mesure d'une performance économique présente). De l'un à l'autre la configuration temporelle diffère tandis que se profile le déplacement ou la coexistence du possible et de l'actuel, le déplacement de la rationalité en valeur vers une rationalité par objectif (selon les termes de M. Weber), d'une activité communicationnelle vers une activité instrumentale (Habermas).

6 Une double institution (communicationnelle-gestionnaire) de la question du développement durable se profile donc et que nous pouvons expliciter ainsi :

7 a- D'un côté apparaît une force utopique et politique : une capacité d'utopie que l'on croyait perdue, un retour de l'idéal alors que l'on croyait révolus ${ }^{1}$ les grands récits et leur thématique libératrice et salvatrice. Misant sur une large ouverture interprétative et suscitant un creuset potentiellement fécond de discussions, d'interprétations et appropriations, d'innovations (produits, sociales, politiques, légales : introduction du principe de précaution), le rapport Bruntland exprime sur un plan international en 1987 une volonté et un possible. Nous repérons dans la notion désormais campée de développement durable toutes les caractéristiques d'une rationalité en valeur, une idée régulatrice, un enjeu de discussion et une occasion de réorganisation des grands choix du vivre ensemble.

b- D'un autre côté, nous sont donnés à lire des documents comptables nouvelle manière, des outils de gestion intégrés dans les stratégies et les tableaux de bord, insérés dans un calcul coûts-bénéfices qui s'énonce, se constate, s'apprécie, se vérifie et se note. Le modèle du rapport financier est ici dominant avec son cortège d'acteurs, de rapports, de mesure et qui constitue une sorte de médiation réduisant la complexité du 
réel, facilitant la prise de décision des investisseurs, livrant ainsi une réponse unique à une multiplicité de questions et de critères possibles.

\section{Le développement durable comme principe de recomposition du temps et des choix}

Un principe de réorientation de la décision. La notion onusienne présentée en 1987 dans le rapport «Our Common Future» est le grand référent, le texte canonique à partir duquel va être développée une large gamme d'interprétations et de citations. Ce terme est d'abord, aspect souvent oublié, un terme issu de la pensée économique qui propose de renouveler la compréhension et les politiques du développement à l'échelle de la planète (rôle de René Passet) et propose de penser l'environnement comme un bien public. Ce texte une réorientation tant spatiale que temporelle: traitement généralisé du problème des besoins à l'échelle de la planète (" les besoins essentiels des plus démunis, à qui il convient d'accorder la plus grande priorité ", extrait du chapitre 2 du rapport), traitement des « besoins du présent sans compromettre la capacité des générations futures de répondre aux leurs ». Une double solidarité est ainsi envisagée : au niveau horizontal (intragénérationnel, engageant les contemporains du nord et du sud de la planète) et vertical (intergénérationnel, entre générations).

10 Se profilent ici un idéal càd un possible comme le laisse entendre la racine obsessionnelle (able-ability, capacité- possibilité) de sustainability (soutenable, durable capable d'être supporté et de durer), une maxime analogue à celle de la mentalité élargie kantienne: intégrer autrui et le point de vue de l'autre dans la conduite de l'action. Est ainsi mis en avant un principe de responsabilité qui prend plusieurs formes, morales, économiques et juridiques : du principe pollueur-payeur (intégré dans le droit européen depuis 1987) au principe de prévention et de précaution (intégré dans le droit français en 1995). Il s'agit ici de prendre en compte ce que les économistes nomment des externalités, de passer de l'externalité (conséquence involontaire d'un acte) à l'internalité, à l'intégration des conséquences de l'action ce qui suppose l'introduction d'une volonté de justice dans un univers qui produit de l'injustice. Se faisant, la problématique du Développement Durable permet d'envisager autre chose que le modèle libéral classique dont le cœur est le business et la périphérie constituée par le hors business pratiqué (dans le meilleur des cas) sous forme de mécénat ${ }^{2}$, activité située à la marge.

11 Notons cependant que l'appropriation sociale dominante du terme de développement durable ne retient que la dimension temporelle et occulte la théorie de l'équité mondiale énoncée dans le rapport Brundtland comme si les descendants (du nord) étaient préférés aux contemporains (du sud).

Un principe d'ordonnancement des choix.

L'ordre terminologique d'apparition des trois composantes (économie viable, environnement vivable, société équitable) du Développement Durable est important, l'ordre chronologique et l'ordre logique se combinent subtilement dans l'énoncé de trois dimensions: social, économique, environnemental. Le mystère leibnizien des compossibles (problème des possibles capables de passer ensemble à la réalité) se retrouve ici ouvrant le problème du choix, suscitant un jeu ouvert, un lieu propice à 
l'activité délibérative, activité politique nouvelle ou complémentaire au principe traditionnel de la représentation.

Sur un plan logique l'articulation économique-environnemental est souvent pensée en termes d'écoefficience à savoir la production de biens et de marchandises à prix compétitifs et maintenant ou augmentant une qualité de vie pour l'individu et la planète (le calcul éco. intègre alors ce que la terre peut supporter). Le couplage environnement -social pose la question de l'équité entre générations présentes (équilibre Nord-Sud) et à venir (des retraites à la question de la biodiversité). Le couplage économique et social pose la question de la justice sociale sur plusieurs plans: contribution/rétribution des salariés dans les entreprises, contribution des entreprises envers les collectivités et sociétés dans lesquelles elle opèrent.

Le problème des intersections entre les deux et à plus forte raison les trois sphères est donc crucial, il engage une réorganisation possible du présent sur la base d'un avenir envisagé. L'ordre de présentation des fameux trois piliers engage des choix humains et politiques différents. Placer la dimension environnementale en première position revient à opérer un choix privilégié sur ce qui est alors envisagé comme un levier du développement économique et social. Est ici en jeu une manière spécifique de traiter l'économique et le social en les subordonnant au principe environnemental, en les irriguant de principes nés autour de la question environnementale tels le principe de précaution qui irradie le processus de prise de décision ou le souci des générations à venir. A l'opposé situer l'environnement en troisième position signifie que la préoccupation environnementale intervient après la satisfaction des besoins fondamentaux (économiques et sociaux, confort et liberté) ce qui lui donne un peu le statut de la cerise sur le gâteau. L'ordre des termes recouvre un vif débat qui oppose les tenants d'un «durable faible» (misant sur l'optimisation des ressources et la modernisation écologique dans le cadre du marché), un «durable fort » (visant la maximisation du bien être social, la limitation des ressources et l'adoption du principe de précaution) voire du "durable très fort " (la vie, critère et fin ultimes rassemble les tenants des théories de la décroissance et de la deep ecology et son cortège d'obligations envers la vie). Certains pays comme le Canada ont, après un long débat et une réflexion concertée, opté pour un classement des trois paramètres sous le registre moyens/fin : la fin suprême énoncée est le développement social (choix anthropocentrique fondamental) et les deux moyens retenus sont l'environnemental et l'économique.

La tension entre les principes du développement durable engage une dimension humaine (la question de l'anthropocentrisme), temporelle et territoriale. Si l'Agenda 21 permet d'envisager le passage et la déclinaison du niveau international au niveau local, les tensions sont vives sur un plan local. Elles se situent entre différents niveaux d'exigence, entre des questions incessantes : faut il sauver l'emploi ou l'environnement (cas des activités polluantes), sauver les résultats et le cours de l'action ou maintenir l'emploi ? C'est dans ce domaine que les tensions sont les plus vives, les discussions les plus fortes, l'écartèlement le plus aigu.

\section{L'approche gestionnaire : vers une technologie d'un idéal et la mise en équation du possible}

Il s'agit ici d'envisager la mesure du Développement Durable et de la construction d'un jugement comptable et de comprendre la technologisation d'une question devenue 
publique, la réduction d'un débat politique à une série d'indicateurs. En un mot il s'agit de comprendre comment le délibératif peut être mis en équation. efforts conjugués des pouvoirs publics et de l'opinion. Les lois de 2002 sur les Nouvelles Régulations Economiques rendent cette pratique obligatoire pour les entreprises cotées au premier marché (soit près de 700 entreprises). L'article 116 de la loi 2001-420 du 15 mai 2001 énonce l'obligation de publicité sur deux points: la gouvernance et la dimension sociale et environnementale de l'activité de l'entreprise. Le rapport annuel doit fournir des informations sur les "conséquences sociales et environnementales " des entreprises cotées sur le premier marché. Le décret d'application spécifique du 20 février 2002 précise la nature des informations qui doivent être publiées.. La loi NRE dont certains estiment qu'elle est la plus contraignante à l'échelle mondiale, demande que soient évaluées et publiées les dépenses environnementales («rejets dans l'air, l'eau et le sol affectant gravement l'environnement, gaz à effet de serre, substances toxiques et radioactives »), elle met l'accent sur la nécessité de publier mais non de certifier ou de vérifier. Parallèlement à la loi, l'information extra financière, de type sociétale est née d'un ensemble d'initiatives nées en réponse ou en anticipation par rapport à des demandes émanant d'acteurs aussi divers que les consommateurs (et leurs associations), les salariés (et leurs syndicats), les ONG, élus et autres publics. Le reporting est une pratique née dans les années 1990, recommandée par l'Agenda 21 cette pratique de la publication d'informations sociales et environnementales a été initiée par des entreprises pionnières telle The Body Shop.

acteurs en présence: Nous retiendrons ici trois niveaux, trois familles d'acteurs intervenant dans la création de dispositifs, de technologies de mesure du Développement Durable. Ces trois familles ne couvrent pas toute la gamme des acteurs intervenant mais nous les avons retenu pour deux raisons: leur travail instituant, normalisant ou producteur de normes et les trois positions qu'ils représentent. Le premier groupe est d'ordre international, il engage des principes et des standards de 
jugement internationaux. Le second représente les groupes de travail et associations créées sur ce point, leur particularité est d'intégrer largement un principe de concertation et de discussion dans la création d'outils de mesure. Les troisièmes sont les agences de notation dans lesquelles la mesure s'effectue sans débat, les choix et indicateurs étant ici solidifiés en une batterie d'indicateurs.

Premier niveau d'intervention, d'impulsion et de mesure, le niveau international, du moins celui des organisations internationales qui édictent non pas des instruments de mesure mais des principes généraux qui sont autant de grands référents. Ces textes d'institutions publiques ou internationales sont jugés incontestables : normes de l'OIT (Organisation Internationale du travail), textes de l'ONU, OCDE ou Union Européenne (cf le Livre vert de la commission européenne intitulé « Promouvoir un cadre européen pour la responsabilité sociale des entreprises »). Ils affirment des valeurs universelles et proposent des instruments de réalisation et de mesure de ces valeurs. C'est ainsi que le GRI (Global Reporting Initiative)a été créé en 1997 en collaboration avec le Programme des Nations Unies pour l'Environnement. Sa mission est d'élaborer et de diffuser les lignes directrices d'un reporting développement durable à l'échelle mondiale, proposition d'un format éditorial et d'un standard de jugement. Cette opération est complémentaire de celle du Global Compact (Pacte Mondial). Une première version fut publiée en 1999, une seconde en 2000 puis une troisième en 2002, signes de la difficulté. L'application de ces lignes repose sur le volontariat. Le Global Reporting Initiative est une initiative internationale destinée à ancrer la publication de rapports environnementaux et sociaux. Lancé en 1997 par un ensemble recouvrant des ONG, des investisseurs, des gestionnaires de fonds éthiques, des organisations syndicales et religieuses, il repose sur le volontariat. Il présente les objectifs, les destinataires et les qualités requises de l'information publiée. Il fonctionne donc comme un guide permettant l'élaboration du rapport. Trois caractéristiques concernent l'information visée : sa délimitation (autour des critères d'exhaustivité et de pertinence: l'exhaustivité renvoie au périmètre du rapport, la pertinence est corrélée aux besoins de l'organisation et de ses publics), sa fiabilité (corrélée à sa précision, sa neutralité et a comparabilité) et son accessibilité (clarté, lisibilité -le contenu doit être intelligible pour tous- et périodicité).

Second niveau, celui de la création et multiplication de groupes de travail visant l'élaboration d'outils de mesure. L'ORSE qui rassemble des syndicats, le groupe de travail «Environnement» créé au sein de l'AFNOR rassemblant des entreprises, des organismes publics, des administrations, des ONG, des syndicats et organisations professionnelles sont représentatives de ces structures dédiées à la question de la mesure de la performance sociétale. Le groupe AFNOR a conçu le guide SD 21000 qui s'adresse aux entreprises de toute taille et de tous secteurs et leur propose une méthodologie d'intégration de la problématique de développement durable dans leur stratégie et leur système de management. Ce guide est composé de deux parties, l'une générale et stratégique concerne la notion de développement durable et ses conséquences sur le plan du fonctionnement des entreprises, la seconde concerne la mise en œuvre concrète de la démarche et propose des questionnements permettant à l'entreprise de situer sa démarche puis un ensemble de recommandations hiérarchisées. Ce texte intègre et reflète la diversité des points de vue des membres, il a été mis en débat via le site afnor.fr durant quatre mois, fin 2002 ce qui a entraîné un remaniement $\mathrm{du}$ texte. Un guide mouvant, en devenir, en construction et reconstruction, un texte normatif sans l'être qui prévoit la place du questionnement 
(contre une logique de l'adaptation pure et simple), qui met l'accent sur l'identification des enjeux et des parties intéressées et qui porte toujours les traces de la discussion dont il est le fruit.

Troisième niveau, les agences de notation extra financière. Aux agences de notation financière évaluant les risques encourus pour toute opération financière s'ajoutent les agences de notation extra-financière, sociales et environnementales crées dans les années 1990-2000. En 1990 est créé le premier indice boursier éthique, le Domini Social Index (DSI 400) créé par Amy Domini qui regroupe 400 firmes sélectionnées sur la base de leurs performances sociales, environnementales et éthiques. Il existe aujourd'hui environ une trentaine d'agences de notation sociétale dans le monde, les plus puissantes étant le Dow Jones Sustainability Index américain et le FTSE4Good britannique qui recouvre une gamme d'indices de responsabilité sociale. L'ASPI Eurozone est un indice mesurant dans la zone euro la performance boursière des sociétés les plus engagées au regard de leur responsabilité sociale et environnementale, l'instrument de mesure est celui de l'agence française Vigéo.

Chaque agence mobilise des indices qui intègrent une description du social et engagent une catégorisation et une qualification des organisations. Chaque référentiel mis en œuvre permet de standardiser une information en résumant les différentes mesures sous une forme considérée comme intelligible et communicable. Il s'agit là d'artefacts qui donnent une certaine représentation, opèrent une schématisation des situations ainsi qu'un gommage des dimensions les plus controversées. Cette stratégie d'écriture est aussi une stratégie de mise en lisibilité et en visibilité des entreprises soumises à notation.

Dans tous les cas, nous assistons à un même travail de découpage «scientifique » de la réalité en fonction de facteurs classés, ordonnés, à l'instauration d'une taxinomie des valeurs animée de façon sourde mais puissante par une guerre méthodologique qui est une guerre des possibles et des souhaitables.

28 La première agence créée en France avec le soutien de la caisse des Dépôts en 1997, Arèse, devient en 2002 Vigéo, dont le capital est tripartite (entreprises, syndicats européens et organismes financiers) est dirigée par une figure emblématique de la vie syndicale, Nicole Notat. La méthodologie mobilisée repose sur six thématiques principales: les ressources humaines, l'environnement, les relations clientsfournisseurs et sous-traitants, le gouvernement d'entreprise, l'engagement sociétal et les droits humains. L'accent est ici mis sur les modes de direction et sur les résultats. Par ailleurs, l'originalité de Vigéo consiste à distinguer les secteurs d'activités, la comparaison entre un distributeur et une compagnie aérienne étant de fait rendue impossible dans cette logique contrairement à bon nombre d'approches anglosaxonnes. La notation finale est un jugement engageant trois qualificatifs : pionnier, moyen ou non concerné. La mesure et le jugement prononcés par Vigéo concernent le volontarisme de l'entreprise, sa capacité à intégrer le moyen et long terme dans sa stratégie et dans ses résultats. Le temps du progrès et des successeurs est la toile de fond de cette approche.

L'agence franco-anglaise Core Ratings est créée en 2002 en réaction à Vigéo, à l'initiative de Geneviève Ferrone évincée d'Arèse. Elle est adossée à Fitch $^{4}$ et fait reposer sa notation de la responsabilité d'entreprise sur l'analyse de la gestion du risque et associe implicitement création de valeur et maitrise du risque. Elle évalue les entreprises sur leur manière d'intégrer et de maîtriser les risques inhérents à leur activité : risques 
environnementaux, sociétaux (impact sur les sociétés où intervient l'entreprise), sociaux (emploi, recrutement, relations de travail, protection sociale) et éthiques. Le lien création de valeur- évaluation des risques est au cœur de la démarche de Core Rating qui propose par ailleurs une notation sur la gouvernance d'entreprise. La temporalité est ici intégrée comme facteur de risque et l'évaluation porte sur la capacité de chaque organisation notée à introduire de la continuité dans une discontinuité fondamentalement présupposée, de la visibilité ou prévisibilité dans un temps conçu comme menaçant ou source de menaces. Le temps de l'incertitude et des contemporains est ici clairement privilégié.

L'agence américaine Innovest créée en 1995 est plus particulièrement dédiée à la politique environnementale des entreprises, à l'examen de leur stratégie et management environnemental. Elle tient compte pour cela des caractéristiques du secteur d'activité. L'écoefficacité est ici privilégiée et se traduit par la création d'un algorithme multi-facteurs, Ecovalue 21. La notation se traduit par des lettres, sans qualificatif explicite allant de AAA (le meilleur) à CCC. Le temps présupposé par cette méthodologie est le temps des successeurs.

31 Chaque agence travaille selon sa propre méthodologie laquelle exprime un choix spécifique, explicite ou non, envers ce qu'est ou devrait être une entreprise, envers sa place et sa responsabilité dans la société et dans le monde. Chaque méthodologie exprime une définition de la performance, une vision du temps et du monde c'est-àdire de la société, du marché et des règles qui les régissent ou devraient les régir. La variété, la multiplicité des méthodologies est essentielle et irréductible.

Des organismes internationaux aux agences de notation via les groupes de travail, nous avons affaire à trois types d'acteurs mais aussi et surtout à trois manières de camper le cadre d'un débat ou de le raccourcir, d'actualiser ou de contracter le temps long: les acteurs internationaux définissent des principes et un cadre (celui d'une mentalité élargie dans la prise de décision), les groupes de travail s'attachent à co-construire des normes (à réguler conjointement présent et avenir selon un principe de faisabilité), les agences de notation fixent une note. Trois manières de dire, de juger, de qualifier sont en présence, mobilisant de manière différente la place du débat et de la délibération. Notons que le troisième groupe d'acteur est celui qui agit de la manière la moins délibérative et la plus "technologique ", la note pouvant être considérée comme le résultat d'une technologie voire comme un média sans langage, cette notion habermassienne renvoyant à l'organisation tacite d'un accord en dehors de toute discussion.

\section{Information contre communication}

Du temps long au temps court du développement durable se jouent le régime de la communication (entendue comme activité discussionnelle) et le statut de l'information. Dans ce domaine, nous sommes conduits à développer une idée : l'inverse proportion entre communication et information, à savoir que moins il y a de communication, plus il y a d'informations. La problématique du développement durable nous semble donner lieu à une profusion d'informations et à peu de débats bien que cet aspect soit à nuancer à la lumière de quelques grands événements tel le Sommet de Johannesburg en 2000. Nous assistons dans ce domaine à une réduction du débat et de l'agon au profit de la production abondante d'informations qui paradoxalement peut faire débat mais en 
fait le tarit. Un volume colossal d'informations est déversé sur ce thème, mobilisant différents médias parfois redondants parfois complémentaires mais ce colosse informationnel a un socle fragile. Cette profusion d'information s'accompagne d'un mythe de la transparence qui produit en fait de l'opacité et une sorte de consensus non voulu, non discuté, quasiment indifférent. La production abondante d'informations capable de susciter et d'entretenir le débat semble en fait le tarir, du moins en atténuer les différends et les formes agonistiques.

\section{Le statut de l'information}

Premier problème : celui du recueil et de la formalisation de l'information. La base de travail et d'informations permettant la notation d'une organisation est la même pour toutes les agences. Elle est constituée de documents publics concernant l'entreprise en notation (documents internes, commentaires et citations dans la presse, position des syndicats, rapports d'ONG, procès intentés ou en cours dans cette entreprise etc), de questionnaires spécifiques, d'entretiens avec les responsables de l'entreprise et les parties prenantes. Dans ces corpus, il convient de souligner la prépondérance des textes et propos déclaratifs qui posent le problème de la qualité et de la fiabilité de ces informations sources du travail de jugement.

Second problème, celui du mode de qualification. Comment formaliser un jugement, entre notes, lettres ou qualificatif que peut on choisir et que convient il de préférer? Comment réduire la complexité du réel ? Ici se jouent la tension entre les principes de simplification et de pertinence de l'information et la difficile mise en équation du social ou de l'organisationnel. La question du format est importante, au départ Arèse présente ses données sou forme d'un livret de 4 à 5 pages mais ces fiches ont été jugées trop longues par des investisseurs qui déclarent crouler sous les données et les chiffres. Les fiches vont alors être réduites et s'intégrer dans un fichier Excel. L'orientation quantitative est clairement privilégiée, ce qui rend par contraste originale et intéressante la position du CFIE qui refuse de quantifier l'évaluation d'une entreprise.

\section{Réorientation de la communication institutionnelle}

Se pose ici un autre problème, celui du lien entre reporting et communication et de l'installation d'une confusion dans les pratiques. La pratique du reporting est une pratique d'évaluation (et donc de détermination d'objectifs de progrès) qui n'est pas forcément assortie d'une publicisation. L'enchaînement entre ces deux aspects est cependant de plus en plus courant, lié à la fois à des obligations légales d'information mais aussi et surtout à une réorientation de la communication institutionnelle des entreprises.

Les rapports environnementaux ou de Développement Durable sont devenus obligatoires dans un certain nombre de pays et concernent les grandes entreprises (généralement cotées). 45\% des multinationales publient un tel rapport et le mouvement s'accélère. Mais la plus grande hétérogénéité s'y constate : la délimitation du périmètre est variable (quelles entités sont incluses dans les données chiffrées, souvent des filiales ne sont pas intégrées pour des raisons objectives ou subjectives), les domaines couverts sont très disparates, les formats et les contenus sont très variés. 
38 Le rapport annuel est un texte qui montre et qui cache à la fois, qui informe mais n'éclaire pas toujours. Les domaines les mieux couverts en France sont dans le domaine social les indicateurs sur la politique de formation des salariés, la consommation d'eau et d'énergie, à l'inverse le plus grand flou ou le silence concerne les relations de l'entreprise avec son environnement, les nuisances et rejets. C'est un document ciblé (destiné aux actionnaires, investisseurs, analystes financiers voire grand public), objet de luxueuse brochure ou accessible en ligne, objet de prix et distinctions qui a par conséquent un rôle de médiation sociale entre les acteurs et de prescription de conduite. Sa crédibilité est donc un point essentiel et l'objet d'âpres recherches de garantie. Des dispositifs de certification ${ }^{5}$ empruntés à l'analyse financière sont mis au point par des organismes spécialisés (tel le cabinet Price Waterhouse Coopers) et reprennent les niveaux d'assurance adoptés par les experts comptables (assurance faible-modérée-raisonnable). Dans cette quête se joue le problème de la confiance et de la présence du tiers exclu ou inclus. Le rapport Danone 2004 publié en 2005 révèle cette quête illimitée et pour asseoir le propos et le garantir mobilise un ensemble de dispositifs destinés à susciter le crédit : le rapport Price Waterhouse Coopers attestant d'une assurance modérée concernant les informations livrées, les notes attribuées par deux agences de notation, l'une française, l'autre internationale (Dow Jones Sustainability index). Il fait de plus intervenir au fil des pages le témoignage des famaux stakeholders en l'occurrence un éleveur allemand, la vice présidente de l'EFSA (Food safety), un médecin de l'OMS, un syndicaliste (secrétaire général de la fédération internationale de l'alimentation) et une ONG ...

Nous terminerons cet exposé par la considération d'une autre forme de communication, publicitaire cette fois qui est confrontée à la même difficulté. La diversité des pratiques et les procès retentissants auxquels elle a donné lieu a conduit la profession à réfléchir sur ce point et à se doter d'un code de bonne conduite. La recommandation «Publicité et développement durable» du BVP (Bureau de Vérification de la Publicité) émise en décembre 2003 constitue une première du genre. La France est le premier et pour l'heure seul pays ayant édicté de tels principes d'autodiscipline destinés à la profession des communicants. Trois principes sont avancés :

40 La véracité: " la publicité ne doit pas tromper le public sur la réalité des actions de l'annonceur en faveur du développement durable, ni sur les propriétés de ses produits et services en la matière $"$.

41 L'objectivité : «l'ampleur de la revendication d'une action en faveur du développement durable doit être proportionnée à l'étendue des actions réellement entreprises. Si l'effort de l'annonceur ne porte que sur un produit/service ou sur un ou des éléments limités, la publicité ne peut exprimer un engagement global.... Toute exploitation d'un argument fondé sur des chiffres, sondages doit pouvoir être justifiée par la détention chez l'annonceur de documents vérifiables, objectifs et actualisés ».

42 La loyauté: " un annonceur ne peut se prévaloir de certaines actions à titre exclusif alors que celles-ci seraient imposées à tous par la réglementation en vigueur... la publicité ne oit pas attribuer à un annonceur l'exclusivité d'une action alors que celle-ci est analogue ou similaire à celle d'autres annonceurs ».

43 L'annonceur devra également veiller à un bon usage des signes, des mots et des couleurs. «L'emploi de notions comme celles de placements éthiques, investissements responsables, bonne gouvernance ou tout autre se réclamant de l'éthique doit pouvoir 
être justifié par le respect de critères précis ", "le choix des signes, des termes et des couleurs ne doit pas suggérer des vertus écologiques que le produit ne posséderait pas ».

\section{BIBLIOGRAPHIE}

Acot P., Histoire de l'écologie, paris, PUF, 1988

Bourg D., Quel avenir pour le développement durable ? Paris, Le Pommier, 2002

Boy D., L'écologie au pouvoir, Paris, Presses de Sciences Po, 1995

Callon M ; Lascoumes P., Barthe Y., Agir dans un monde incertain, Paris, Seuil, 2001

D’Almeida N., Les cahiers du GRIPIC : Enjeux et acteurs du développement durable (juin 2003), Dire le

développement durable (juin 2004), Communication durable, communication paradoxale (juin 2005)

Dubigeon O., Mettre en pratique le développement durable, Paris, le Village Mondial, 2002

Gayte O., Conception des systèmes d'information sur l'environnement, Hermès, 1997

Maréchal J.P (ed), Le développement durable : une perspective pour le XX ${ }^{e}$ siècle, Rennes, Presses

Universitaires de Rennes, 2005

Revue Autrement, Les nouveaux utopistes du développement durable, Paris, 2002

Sen A., Development as freedom, New York, Anchor Books, 1999.

\section{NOTES}

1. J.F Lyotard, La condition postmoderne, Paris, Minuit, 1973

2. Social, environnemental, culturel ou sous toute autre forme

3. Le cadre américain est organisé autour de la SEC, créée en 1933 et qui a pour mandat de veiller à ce que les entreprises agissent de manière responsable vis-à-vis des actionnaires lesquels doivent disposer d'informations financières et comptables fiables et transparentes. C'est une institution de la confiance née après le Krach de 1929,elle dresse un cadre qui ne changera quasiment pas jusqu'à l'adoption de la loi Sarbanes Oxley. Cette dernière adoptée en juillet 2002 fait suite aux scandales Enron et WorldCom, elle concerne toutes les sociétés cotées sur le marché nord américains auprès de la SEC et impose aux directeurs généraux et directeurs financiers d'engager leur responsabilité sur la fiabilité des informations communiquées. Les trois grands principes de cette loi concernent l'exactitude et l'accessibilité de l'information, la responsabilité des gestionnaires et l'indépendance des certificateurs-auditeurs.

4. Troisième agence mondiale de notation financière

5. Selon l'ORSE seules $28 \%$ des entreprises cotées ont fait vérifier la fiabilité de leurs infos environnementales et sociales par un tiers 


\section{AUTEUR}

\section{NICOLE D'ALMEIDA}

Nicole d'Almeida est Professeure à l'Université de Paris Sorbonne (Celsa), chercheur au GRIPIC. Mail : nicole.dalmeida-celsa@paris4.sorbonne.fr 